\title{
BMJ Open The influence of narrative medicine on medical students' readiness for holistic care practice: a realist synthesis protocol
}

\author{
Yufrica Huang, ${ }^{\oplus 1}$ Lynn V Monrouxe, ${ }^{\oplus 2}$ Chien-Da Huang ${ }^{1,3}$
}

To cite: Huang Y, Monrouxe LV, Huang C-D. The influence of narrative medicine on medical students' readiness for holistic care practice: a realist synthesis protocol. BMJ Open 2019;9:e029588. doi:10.1136/ bmjopen-2019-029588

- Prepublication history and additional material for this paper are available online. To view these files, please visit the journal online (http://dx.doi org/10.1136bmjopen-2019029588).

Received 01 February 2019

Revised 11 July 2019

Accepted 12 July 2019
Check for updates

(C) Author(s) (or their employer(s)) 2019. Re-use permitted under CC BY-NC. No commercial re-use. See rights and permissions. Published by BMJ.

${ }^{1}$ Chang Gung Medical Education Research Center, Chang Gung Memorial Hospital Linkou Branch, Taoyuan, Taiwan ${ }^{2}$ Faculty of Health Sciences, The University of Sydney, Sydney, New South Wales, Australia

${ }^{3}$ Medical Education and Thoracic Medicine of Chang Gung

Memorial Hospital, Chang Gung University College of Medicine, Taoyuan, Taiwan

Correspondence to

Dr Chien-Da Huang;

cdhuang@adm.cgmh.org.tw

\section{ABSTRACT}

Introduction Holistic healthcare considers the whole person - their body, mind, spirit and emotions_-and has been associated with narrative medicine practice. Narrative medicine is medicine performed with narrative skill and has been offered as a model for humanism and effective medical practice. Narrative medicine interventions have been associated with physicians' increased empathy and more meaningful interactions with patients about managing their illness and preventative medicine. However, while there is some evidence that certain groups are more open to narrative practices (eg, traditional vs Western medical students), the extent to which narrative medicine interventions during undergraduate medical education impacts on students' readiness for holistic care, as well as the underlying reasons why, is unknown.

Methods and analysis Realist review is a theory-driven approach to evaluate complex interventions. It focuses on understanding how interventions and programmes work (or not) in their contextual setting. This realist synthesis aimed to formulate a theory around the influence of narrative medicine medical students' readiness for holistic care practice. We will follow Pawson's five steps: locate existing theories, search strategy, study selection, data extraction, data analysis and synthesis. We will use the following electronic databases: Web of Science, Medline, Scopus and Embase. Articles between January 2008 and September 2018 will be included. Results will be written according to the RAMESES (Realist And Meta-narrative Evidence Syntheses: Evolving Standards) standard for reporting realist syntheses.

Ethics and dissemination Ethics approval was obtained from the Chang Gung Memorial Hospital for the wider study. The findings of this review will provide useful information for academics and policymakers, who will be able to apply the findings in their context when deciding whether and how to introduce narrative medicine programmes into medical students' curricula We will publish our findings in peer-reviewed journals and international conferences.

PROSPERO registration number CRD42018115447.

\section{BACKGROUND}

Holistic healthcare is a form of healing that considers the whole person-body, mind, spirit and emotions-in the quest for optimal health and wellness. ${ }^{12}$ It is essentially

\section{Strengths and limitations of this study}

This study is one of the first to examine preparedness for holistic care as an outcome to narrative medicine interventions.

- The use of a systematic approach to identifying the literature around outcomes relating to holistic care arising from narrative medicine interventions is a study strength.

- The application of a realist approach to understanding the contexts in which narrative medicine prepares different types of students for holistic care practice, and how, is another strength.

- One concern for this study is that there might be a limited number of studies that have examined holistic care and its associated components as an outcome to narrative medicine interventions.

- One further concern is that the reporting of narrative medicine intervention outcomes might predominately focus on reactions to the intervention rather than provide deeper understanding of the mechanisms that might promote/inhibit holistic care.

synonymous with Engel's biopsychosocial model. $^{3} \quad$ The biopsychosocial approach to illness comprises four systems within the person: the organs, the whole person, behaviour and social roles. There are also four contextual factors that influence these systems: personal factors, physical environment, social environment and time. ${ }^{2}$ Holistic care asserts that the patient is a person, not a disease. Thus, treatment involves treating the underlying cause of the condition rather than just alleviating the symptoms. ${ }^{12}$

Recent research has identified individual attributes of clinicians that are optimal for providing holistic care. For example, key personal attributes such as sociability, compassion, respectfulness, patient centredness and sensitivity are all thought to facilitate holistic care provision. ${ }^{4}$ Furthermore, being able to identify and to satisfy patients' needs has also been identified as a motivational factor that enable healing relationships with patients to develop, thereby encouraging an holistic 
care approach. ${ }^{4}$ Finally, having the foresight and ability to facilitate autonomy and self-confidence in patients, to support individuals in obtaining relevant information about their condition and to enhance effective communication all contribute towards individuals' sense of empowerment around making medical treatment decisions. ${ }^{56}$ As such, providing holistic care means understanding how an illness affects the whole person and how to respond to their specific needs. ${ }^{7}$

However, the development of a holistic approach to care is not straightforward. For example, in recent years, medical schools across the world have become increasingly concerned around the issue of empathy decline in their students, ${ }^{8}$ especially during the clinical years. ${ }^{10}$ This is possibly due to students' reactions to so-called professionalism dilemmas: situations in which medical students witness or participate in something they believe to be unethical, unprofessional or "wrong'. 1112 Common professionalism dilemma events for healthcare students that give rise to conflicts between their formal professionalism learning and what they witness during work-based placements include student abuse, patient dignity and safety issues. ${ }^{12}{ }^{13}$ While experiencing such situations may lead some students to strongly reject these negative role models, it can also lead to diminishing empathy and professional identity disruption. ${ }^{14}{ }^{15}$ Thus, medical schools are seeking ways to design more effective curricula to cultivate positive character development and professionalism in their students. Indeed, more broadly, the medical humanities, which includes narrative medicine, has been heralded as a remedy to experiences of negative role modelling and has been thought to facilitate compassionate care. ${ }^{16-18}$

\section{Narrative medicine and holistic care}

According to Rita Charon, a major proponent of narrative medicine, narrative medicine refers to clinical practice that is fortified by a narrative competence. ${ }^{19-21}$ Narrative medicine is thought to enhance the attributes of healthcare providers to facilitate the delivery of holistic care practice. In particular, it has been promoted as a way for physicians to understand the personal connections between themselves and their patients ${ }^{20}$; to help them to recognise, interpret and be moved to action by the problems of others ${ }^{21}$; and to provide new opportunities for greater learning about respectful, empathic and nourishing medical care. ${ }^{22-24}$ The narrative concept therefore has been advocated as a framework for practice and proposed ideal (holistic) care while providing the means to gain competence. It is unsurprising therefore that medical schools around the world have introduced narrative medicine as part of their medical humanities programmes in their undergraduate curricula. ${ }^{18}$

Evidence for the benefit of narrative medicine interventions suggests that it can enhance empathy, observational skills, emotional awareness, communication skills, deepen critical thinking and reflective practice, and other factors associated with holistic care. ${ }^{182526}$ Furthermore, a systematic review of the literature on narrative medicine has found the outcomes for patients to be efficacious in terms of decreasing pain; increasing well-being (related to illness), confidence and cooperation; and decreasing stress and feelings of alienation. ${ }^{27}$ Additionally, narrative medicine educational interventions are not always efficacious. Indeed, recent research has begun to unpack the differential engagement and outcomes across study cohorts. For example, when considering the outcomes of a narrative medicine course in Asia, students on a Chinese medicine track reported greater emotional, reflective and self-development outcomes in comparison with students on a Western medicine track. ${ }^{28}$

As we can see, despite the appearance of a link between the desired outcomes of a narrative medicine course and requirements for holistic care practice, evidence is inconclusive. Additionally, to date, no direct evidence unpacking the underlying processes for this potential link has been provided; thus, prior research draws on elements of holistic care to make their assertions (eg, empathy) without illuminating the contexts and mechanisms through which this might have come about. Therefore, understanding the underlying mechanisms that enhance such an outcome of narrative medicine programmes, alongside the necessary conditions for them doing this, is crucial for curriculum designers (the beneficiaries of this research).

To our knowledge, this is the first systematic review to focus on the impact of a narrative medicine intervention on medical students' preparedness for holistic care, with the explicit aim of unpacking the 'black box' of the intervention itself, by asking the following broad research question: under what circumstances and for whom does a narrative medicine intervention in an undergraduate medical curriculum influence medical students' readiness for holistic care?

\section{REALIST REVIEW METHODOLOGY}

Realist review is a theory-driven approach to evaluate complex interventions that focus on understanding how interventions and programmes work (or do not work) in their contextual setting; so, rather than simply measuring outcomes, it explains why interventions work. ${ }^{29-32}$ Standard systematic reviews focus on measuring and reporting on the effectiveness of a programme, but provide little or no clues as to why the intervention works or not when applied in different contexts, deployed by different stakeholders or used for different objectives. ${ }^{33}$ Thus, realist reviews attempt to explain 'How does it work?', 'Why does it work?', 'For whom does it work?' and 'In what circumstances does it work? ${ }^{33}$ Furthermore, standard reviews follow a relatively straightforward formula whereby databases are searched systematically in a uniform manner. However, realist reviews have an iterative approach to searching the literature: having developed an initial search of the core literature, further searches of other literature can be undertaken in the pursuit of other 'lines of enquiry. ${ }^{34}$ 
The hallmark of a realist methodology is the generative model of causality: to infer the outcome (s) $(\mathrm{O})$, there is a need to understand the underlying mechanism (M) that connects to the context $(\mathrm{C})$ in which the intervention occurs. ${ }^{33}$ Realist methodology does not assume a linear causal relationship but attempts to explain complex interventions through programme theory. ${ }^{35}$ As the name suggests, it is an approach grounded in realism, ${ }^{29}$ a school of philosophy asserting that both the material and the social worlds are 'real', that they can have real effects on stakeholders and that it is possible to work towards a closer understanding of what causes change. Realist methodology belongs to a family of theory-based evaluation approaches. It is used to evaluate the impact of an intervention through three key elements and their complex interactions: the context in which reality unfolds, the mechanisms that trigger the outcome following the intervention (or $\mathrm{C}-\mathrm{M}-\mathrm{O}$ model). ${ }^{32}$

There are, of course, limitations to realist reviews. For example, it is intellectually challenging and there is no simple 'formula' as with more traditional systematic reviews. It also requires advanced theoretical understanding drawn from the social sciences, and competencies to design research questions suitable for a context-mechanism-outcome analysis. ${ }^{29-32}$ Despite these limitations, we believe that a realist methodology can facilitate our understanding of the interplay between contexts and mechanisms that might facilitate or inhibit students' readiness to undertake holistic care (the desired outcome).

\section{MAIN RESEARCH QUESTION}

The research question in this study is as follows: what are the contextual factors (including traditional and Western medicine contexts) of narrative medicine interventions and the underlying mechanisms that impact on medical students' readiness for holistic care practice?

\section{REVIEW AIM AND OBJECTIVES}

The study aimed to identify the impact of narrative medicine interventions during undergraduate medical curricula on medical students' readiness to deliver holistic care in order to develop a programme theory (a theoretical model) of what works, for whom and why.

Objectives:

1. To explore how a narrative medicine intervention can facilitate medical students' readiness for holistic care.

2. To develop a programme theory that explains how narrative medicine interventions can facilitate holistic care.

\section{METHODS}

The study design was based on Pawson's five stages (figure 1). ${ }^{33}$

\section{Stage 1: locate existing theories}

We will begin by identifying the relevant theories associated with narrative medicine and its influence on

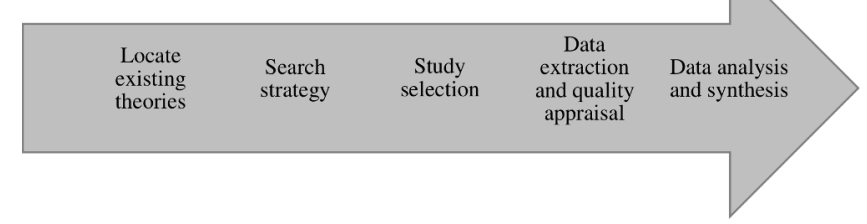

Figure 1 Five stages of a realist synthesis study design (from Pawson).

holistic care practice to develop our initial programme theory around how narrative medicine might influence students' readiness for holistic care practice. This stage involves identifying potential theories by searching the relevant literature to facilitate our understanding and theorising about how narrative medicine might influence students' readiness for holistic care practice in different contexts. This involves a search using electronic published resources (Web of Science, Medline, Scopus and Embase), as well as books. The search will comprise a scoping search, which will be developed using search terms focused on the intervention (eg, narrative medicine, narrative-based medicine, narrative medical, narrative training and parallel charts) and the outcome (eg, preparedness or readiness for holistic care and attributes of holistic care practitioners). Books and articles will be examined, and any identified theories will be used to build up the initial programme theory. This initial theory will be examined against the studies included in the review. This stage has already begun, and so far we have identified the biopsychosocial theoretical perspective and are examining research around facilitators and barriers to becoming biopsychosocial.

\section{Step 2: search strategy}

The second stage involves developing our search strategy that will essentially comprise two phases. We will begin by searching the Web of Science, Medline, Scopus and Embase databases to find relevant articles for the study. The search terms will be developed, tested iteratively and discussed across the research team (see online supplementary appendix 1 for our initial progress). During the second phase of searching, we will seek additional relevant documents for testing and refinement of our programme theory, which may come from grey literature (eg, policy documents, conference proceedings and other works not necessarily subjected to peer review).

\section{Step 3: study selection}

During the searching process, titles and abstracts will be imported to EndNote and screened using the inclusion and exclusion criteria below.

Inclusion criteria:

- Date range: articles between 1 January 2008 and 10 September 2018.

- Population: medical students (clerks and interns) and medical teachers (trainers and educators). 
- Focus: narrative medicine interventions, holistic care (and its components) and patient centredness.

- Outcome: holistic care practice (and its components).

- Language: English and Mandarin.

- Geographic location: any.

Exclusion criteria:

- Date range: articles outside our date range.

- Population: other healthcare students, other healthcare teachers, non-healthcare students and non-healthcare teachers.

- Focus: other medical humanities aspects, narrative data outside of narrative medicine interventions.

- Language: other than English and Mandarin.

- Geographic location: no exclusions.

\section{Step 4: data extraction and quality appraisal}

In realist reviews, data extraction of the selected studies comprises a number of phases. First, we will use a data extraction form to record study details: basic information (author, title and year of publication), document details (aim, design, method and findings), population and intervention. ${ }^{35}$ At this point, we will take our selection of articles for the programme theory development and appraise them for their relevance and rigour, marking them up as conceptually rich (high), moderate and low. All documents that are deemed to contribute to theory testing and refinement will also be assessed for credibility and trustworthiness. ${ }^{36}$ Here we will consider the quality of arguments and theory use, not just at the level of the data, which will enable us to draw on relevant manuscripts for our programme theory development. ${ }^{37}$

Following this, we will identify initial contexts, mechanisms and outcomes for the programme theory development. This will be undertaken in collaboration with the team. Each team member will read a subset of the articles individually before discussing our individual findings in a group. A list of contexts, mechanisms and outcomes will be developed, with full descriptions. All data (identified articles) will be imported into the software ATLAS.ti V.8 and coded accordingly. New contexts, mechanisms and outcomes will be developed throughout this process as and when they are identified.

All data extraction will be undertaken by one reviewer, and the extracted data will be reviewed by the other team members regularly. Any differences in opinions will be discussed during project team meetings and agreements on any new codes will be made together.

We will follow the Preferred Reporting Items for Systematic Reviews and Meta-Analyses guidelines to improve the conduct of systematic reviews and the quality of the protocol (see online supplementary appendix 2).

\section{Step 5: data analysis and synthesis}

Data analysis from step 4 will be synthesised to refine the programme theory, which will identify the contexts and mechanisms that are key for students' readiness for holistic care practice, highlighting what works for whom and why. Specifically, we will infer the mechanisms that trigger the desired outcomes. ${ }^{35} 38$

These findings will be systematically considered in order to test and refine the programme theory using the following conceptual tools ${ }^{39}$ :

- Juxtaposing: when the study provides process data to understand the outcome model mentioned in another study.

- Reconciling: identification of the differences between contradictory sets of findings.

- Adjudicating the data: quality consideration between research.

- Consolidating: inference of a mechanism for a different outcome.

- Situating: explanation of differing outcomes of intervention and completion of the context-mechanismoutcome configurations.

The results of the synthesis will be written according to RAMESES standards for reporting realist syntheses. ${ }^{36}$

\section{Patient and public involvement}

This protocol is a systematic review to focus on the impact of a narrative medicine intervention on medical students' preparedness for holistic care; thus, this research did not involve patients and public involvement.

\section{Ethics and dissemination}

Ethical approval for the wider study (including qualitative interviews at stage 2, not included in this protocol) was obtained from Chang Gung Memorial Hospital (201 601 857B0C601). This study will draw from published literature to describe context-mechanism-outcome configurations regarding how narrative medicine interventions impact on medical students' readiness for holistic care practice. By identifying the causal mechanisms around the influence of narrative medicine interventions on holistic care practice readiness, it may be possible to design narrative medicine programmes that are effective for specific medical students across different cultural and organisational/curriculum contexts. The findings of this review will be submitted for publication to key medical education journals, core international medical education conferences, as well as offered for download as a 'top tips' resource via our research centre website in order to provide useful information for academics and policymakers, who will be able to apply the findings in their context for the improvement of medical students' learning.

Acknowledgements We thank Jan Illing for her methodological advice and review of the manuscript.

Contributors The review was conceived by LVM and C-DH. Data extraction was carried out by $\mathrm{YH}$, with support from LVM and C-DH. YH wrote the first draft of the manuscript with comments and review by LVM. All authors contributed to the revision of the manuscript and approved the final version.

Funding This study was supported by Chang Gung Memorial Hospital, Taiwan (grant number CDRPG 3G0061).

Competing interests None.

Patient consent for publication Not required. 
Provenance and peer review Not commissioned; externally peer reviewed.

Data availability statement There are no data in this work.

Open access This is an open access article distributed in accordance with the Creative Commons Attribution Non Commercial (CC BY-NC 4.0) license, which permits others to distribute, remix, adapt, build upon this work non-commercially, and license their derivative works on different terms, provided the original work is properly cited, appropriate credit is given, any changes made indicated, and the use is non-commercial. See: http://creativecommons.org/licenses/by-nc/4.0/.

\section{REFERENCES}

1. WebMD. What is holistic medicine? secondary what is holistic medicine? 2017. Available: http://www.webmd.com/balance/guide/ what-is-holistic-medicine

2. Wade DT. Holistic health care: what is it, and how can we achieve it? 2009:1-35.

3. Engel GL. The clinical application of the biopsychosocial model. Am J Psychiatry 1980;137:535-44.

4. Zamanzadeh V, Jasemi M, Valizadeh L, et al. Effective factors in providing holistic care: a qualitative study. Indian J Palliat Care 2015;21:214-24.

5. Suhonen R, Valimaki M, Katajisto J. Developing and testing an instrument for the measurement of individual care. $J$ Adv Nurs 2000;32:1253-63.

6. McCarthy V. Freeman, L. H. a multidisciplinary concept analysis of empowerement: implications for nursing. Journal of Theory Construction \& Testing 2008;12:68-74.

7. Mead N, Bower P, Bowe PP-centredness. Patient-centredness: a conceptual framework and review of the empirical literature. Soc Sci Med 2000;51:1087-110.

8. Triffaux J-M, Tisseron S, Nasello JA. Decline of empathy among medical students: Dehumanization or useful coping process? L'Encéphale 2019;45:3-8.

9. Neumann M, Edelhäuser F, Tauschel D, et al. Empathy decline and its reasons: a systematic review of studies with medical students and residents. Academic Medicine 2011;86:996-1009.

10. Hojat M, Vergare MJ, Maxwell K, et al. The devil is in the third year: a longitudinal study of erosion of empathy in medical school. Academic Medicine 2009;84:1182-91.

11. Monrouxe LV, Rees CE. Healthcare professionalism: Improving practice through reflections on workplace dilemmas: Wiley Blackwell, 2017.

12. Monrouxe LV, Rees CE, Dennis I, et al. Professionalism dilemmas, moral distress and the healthcare student: insights from two online UK-wide questionnaire studies. BMJ Open 2015;5:e007518-e18.

13. Rees CE, Monrouxe LV, McDonald LA, Narrative MLA. Narrative, emotion and action: analysing 'most memorable' professionalism dilemmas. Med Educ 2013;47:80-96.

14. Monrouxe L, Shaw M, Rees C. Antecedents and consequences of medical students' moral decision making during professionalism dilemmas. AMA journal of ethics 2017;19:568-77.

15. Rees CE, Monrouxe LV. Who are you and who do you want to be? key considerations in developing professional identities in medicine. Medical Journal of Australia 2018;209:202-3.

16. Friedman LD. The precarious position of the medical humanities in the medical school curriculum. Academic Medicine 2002;77:320-2.

17. Bleakley A, Marshall R, Brömer R. Toward an aesthetic medicine: developing a core medical humanities undergraduate curriculum. $J$ Med Humanit 2006;27:197-213.
18. Chiavaroli N, Huang CD, Monrouxe LV. Chapter 16 Learning medicine with, from and through the humanities. In: Tim Swanwick KF, O'Brien $\mathrm{B}$, eds. Understanding medical education: evidence, theory and practice. 3rd ed. West Sussex: Wiley Blackwell, 2018.

19. Reading CR. Writing, and doctoring: literature and medicine. American Journal of the Medical Sciences 2000;319:285-91.

20. Charon R. The patient-physician relationship. narrative medicine: a model for empathy, reflection, profession, and trust. JAMA : the journal of the American Medical Association 2001;286:1897-902.

21. Charon R. Narrative medicine: form, function, and ethics. Ann Intern Med 2001;134:83-7.

22. Brady DW, Corbie-Smith G, Branch WT. "What's important to you?" The use of narratives to promote self-reflection and to understand the experiences of medical residents. Ann Intern Med 2002;137:220-3.

23. Gaver A, Borkan JM, Weingarten MA. Illness in context and families as teachers: a year-long project for medical students. Academic Medicine 2005;80:448-51.

24. DasGupta S, Charon R. Personal illness narratives: using reflective writing to teach empathy. Academic Medicine 2004;79:351-6.

25. Yang $\mathrm{N}$, Xiao $\mathrm{H}$, Cao $\mathrm{Y}$, et al. Does narrative medicine education improve nursing students' empathic abilities and academic achievement? A randomised controlled trial. J Int Med Res 2018;46:3306-17.

26. Wieżel I, Horodeńska M, Domańska-Glonek E, et al. Is There a Need for Narrative Medicine in Medical Students' Education? A Literature Review. Medical Science Educator 2017;27:559-65.

27. Fioretti C, Mazzocco K, Riva S, et al. Research studies on patients' illness experience using the narrative medicine approach: a systematic review. BMJ Open 2016;6:e011220.

28. Huang C-D, Liao K-C, Chung F-T, et al. Different perceptions of narrative medicine between Western and Chinese medicine students. BMC Med Educ 2017;17:85.

29. Wong G, Greenhalgh T, Westhorp G, et al. Realist methods in medical education research: what are they and what can they contribute? Med Educ 2012;46:89-96.

30. Pawson R, Tilley N. Realistic evaluation. London: Sage, 1997.

31. Wong ELY, Yeoh E-kiong, Chau PYK, et al. How shall we examine and learn about public-private partnerships (PPPs) in the health sector? realist evaluation of PPPs in Hong Kong. Soc Sci Med 2015;147:261-9.

32. Westhorp G. Realist impact evaluation: an introduction. methods lab: overseas development Institute, the Australian department of foreign Affairs and trade (DFAT) and BetterEvaluation, 2014.

33. Pawson R, Greenhalgh T, Harvey G, et al. Realist review - a new method of systematic review designed for complex policy interventions. J Health Serv Res Policy 2005;10(1 suppl):21-34.

34. Booth A, Wright J, Briscoe S. Scoping and searching to support realist approaches. In: Emmel N, Greenhalgh J, Manzano A, eds. Doing realist research. London: Sage, 2018.

35. Weetman K, Wong G, Scott E, et al. Improving best practice for patients receiving hospital discharge letters: a realist review protocol. BMJ Open 2017;7:e018353.

36. Wong G, Greenhalgh T, Westhorp G, et al. RAMESES publication standards: realist syntheses. BMC Med 2013;11:21.

37. Wong G. Gathering in Realist Review. In: Emmel N, Greenhalgh J, Manzano A, et al, eds. Doing realist research. Los Angeles, London, New Delhi, Singapore,Washington DC, Melbourne: Sage, 2018.

38. Brennan N, Bryce M, Pearson M, et al. Understanding how appraisal of doctors produces its effects: a realist review protocol. BMJ Open 2014:4:e005466.

39. RAMESES. The RAMESES projects, 2013. Secondary The RAMESES projects. Available: http://www.ramesesproject.org/ 